\title{
Content- and Task-Specific Dissociations of Frontal Activity during Maintenance and Manipulation in Visual Working Memory
}

\author{
Harald M. Mohr, ${ }^{1}$ Rainer Goebel, ${ }^{2}$ and David E. J. Linden ${ }^{1,3,4}$ \\ ${ }^{1}$ Laboratory for Neurophysiology and Neuroimaging, Department of Psychiatry, Johann Wolfgang Goethe-Universität, D-60590 Frankfurt am Main, \\ Germany, ${ }^{2}$ Department of Cognitive Neuroscience, Faculty of Psychology, Maastricht University, 6200 MD Maastricht, The Netherlands, ${ }^{3}$ Max Planck \\ Institute for Brain Research, D-60528 Frankfurt am Main, Germany, and ${ }^{4}$ School of Psychology, University of Wales, Bangor LL572AS, United Kingdom
}

\begin{abstract}
Working memory, the short-term maintenance and manipulation of information, relies strongly on neural activity in the frontal cortex. Understanding the functional role of this activity is a prerequisite for the understanding of cognitive control mechanisms. Functional imaging studies in human participants have attempted to reveal neural correlates of the subdivision of visual working memory into different processes (maintenance vs manipulation) and according to the type of memorized content. Here, we show, using functional magnetic resonance imaging, a content-specific dissociation of frontal activity, with dorsal premotor areas supporting both maintenance and manipulation of spatial features and more ventral areas supporting maintenance and manipulation of color. Manipulation-specific activity was observed in the anterior middle frontal gyrus, the inferior frontal junction, and the inferior parietal lobe bilaterally. These areas have been implicated in cognitive control, and their activation by the manipulation task conforms to the demand on central executive resources in this condition. We suggest that the enhanced demand on cognitive resources in manipulation compared with maintenance was met by interplay of content- and task-specific modules in a frontoparietal network.
\end{abstract}

Key words: visual; memory; fMRI; spatial; prefrontal; premotor

\section{Introduction}

Visual working memory (VWM) is a system for short-term maintenance and manipulation of information (Baddeley and Hitch, 1974). The lateral prefrontal cortex has been shown to play a crucial role in VWM (Fletcher and Henson, 2001; Curtis and D'Esposito, 2003). Based on electrophysiological recording, lesion, and anatomical connectivity studies in nonhuman primates, it has been proposed that a differential activation of lateral prefrontal cortex with a preponderance of more dorsal or ventral components follows the separation of the posterior visual areas into a dorsal ("where") and ventral ("what") pathway (GoldmanRakic, 1987). According to this "domain-specific" account, the dorsolateral prefrontal cortex (DLPFC) and/or dorsal premotor cortex (PMC) would deal with spatial features and location (Courtney et al., 1998b; Munk et al., 2002) and the ventrolateral prefrontal cortex (VLPFC) with object (Courtney et al., 1998a; Munk et al., 2002) and color information (Elliott and Dolan, 1998; Mohr et al., 2003; Yee et al., 2003). A similar dissociation has also been described for the auditory domain (Rämä et al., 2004). The "process-specific" account of the functional subdivi-

\footnotetext{
Received Nov. 10, 2004; revised Feb. 27, 2006; accepted March 13, 2006

This work was supported by Wellcome Trust Grant 077185/Z/05/Z. We thank Carmen Morawetz for help with data acquisition and analysis and Dr. Paul Downing and Prof. Wolf Singer for comments on a previous draft of this manuscript.

Correspondence should be addressed to David E. J. Linden at the above address. E-mail: d.linden@bangor.ac.uk. DOI:10.1523/JNEUROSCI.5232-05.2006

Copyright $\odot 2006$ Society for Neuroscience $\quad 0270-6474 / 06 / 264465-07 \$ 15.00 / 0$
}

sions in frontal cortex states that the VLPFC is involved in the maintenance of information, whereas DLPFC activity is selectively associated with the manipulation of information (D'Esposito et al., 1999; Owen et al., 1999) or the generation of behavioral plans (Hoshi and Tanji, 2004). These two models are not necessarily mutually exclusive. Curtis and D'Esposito (2003) suggest that whereas maintenance of different types of information occurs in specific frontal premotor areas [superior frontal sulcus for spatial and Brodmann area (BA) for verbal rehearsal], the role of the DLPFC/middle frontal gyrus (46/9) would be domain independent. The latter region has indeed been suggested to be involved in mechanisms of top-down control of working memory (WM) storage, such as selection (Rowe et al., 2000; Rowe and Passingham, 2001) or monitoring (Petrides, 2000).

Previous functional imaging studies used mostly object or face recognition paradigms to test domain-specific organization. In some of these studies, a dissociation of spatial and nonspatial domains might have been obscured by the use of spatially complex visual material to probe the ventral stream (Sala et al., 2003). We aimed to engage the ventral and dorsal pathway selectively by asking participants to memorize the color or the spatial orientation of isoluminant stimuli. Moreover, although most previous functional imaging studies of WM contrasted either different processes or different types of material, our experimental paradigm allowed us to assess both domain and process specificity in the same subjects and with the same visual material. This was achieved by designing a color and spatial manipulation task that 


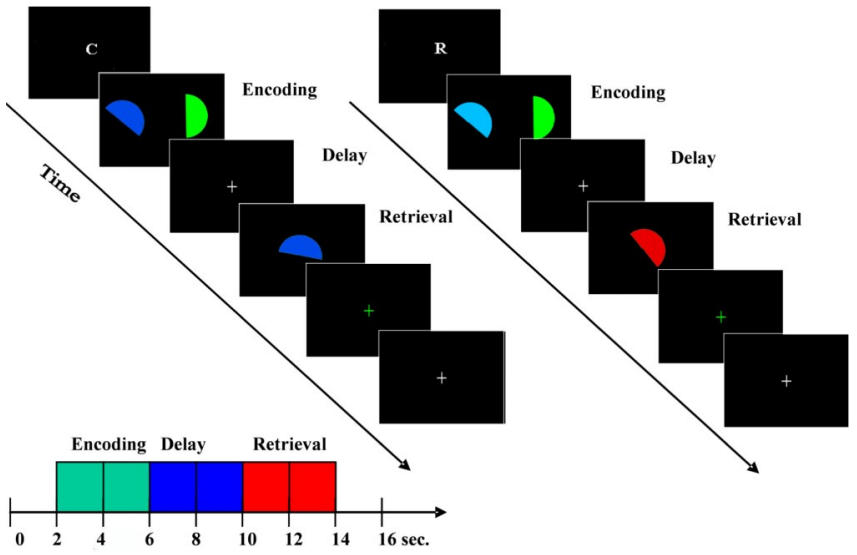

Figure 1. A, Trial in experiment 1. Instruction, $1500 \mathrm{~ms}$; gap, $500 \mathrm{~ms}$; sample presentation, $500 \mathrm{~ms}$; delay, $7500 \mathrm{~ms}$; test presentation, $3000 \mathrm{~ms}$; feedback by change of the color of the fixation cross, $1000 \mathrm{~ms}$; intertrial interval, $6000 \mathrm{~ms}$ (white fixation cross). Task, Subjects had to maintain in WM the color (C) or rotation angle of both half-circles (R). $B$, Experiment 2 had the same time structure and stimuli as experiment 1. One test stimulus was presented ( $3000 \mathrm{~ms}$ ). Task, Subjects had to mix color (C) or rotate the half-circle (R). C, The three task phases of experiments 1 and 2 : encoding ( 2 volumes), delay (2), and retrieval (2). This model ensured that delay activity was relatively uncontaminated by stimulus-driven activity.

required subjects to mix the colors of two probe stimuli or to compute the intermediate angle during the memory delay. We documented with a dual-task-design that the color and spatial tasks indeed rely on different resources in working memory and that the maintenance and manipulation tasks differ in the extent to which they require central executive resources (Mohr and Linden, 2005).

\section{Materials and Methods}

Participants. Thirteen right-handed subjects with normal sight and color vision (Ishihara, 2001) participated in experiments 1 and 2. Visual stimuli were controlled by a personal computer that was triggered by the magnetic resonance (MR) tomograph. They were presented with a liquid crystal display projector (LC-6000; Eiki, Idstein, Germany) with dark background $\left(0.26 \mathrm{~cd} / \mathrm{m}^{2}\right)$, which was calibrated with a Konica Minolta Holdings (Tokyo, Japan) CS-100 photometer.

Memory tasks. In the maintenance task, subjects had to memorize two colored semicircles (Fig. $1 \mathrm{~A}$, see timing information). After the retention interval, they were presented with a test stimulus and had to decide whether it matched the sample in color (color condition) or orientation (spatial condition). In the manipulation task (Fig. $1 B$ ), subjects memorized similar semicircles but, in addition, had to compute the intermediate angle or color. At test, they had to decide whether the probe stimulus matched this intermediate angle (spatial condition) or color (color condition). We demonstrated previously, using a dual task design, that the angle and color tasks can be performed without interference and concluded that they rely on segregated cognitive mechanisms (Mohr and Linden, 2005).

The color maintenance and manipulation tasks could not be solved verbally, because similar colors were used for the matching and nonmatching test stimulus. Consequently, we did not find any use of verbal recoding in the color and orientation maintenance and manipulation tasks under articulatory suppression in previous behavioral experiments (Mohr and Linden, 2005). The maintenance and manipulation tasks differed in the extent to which they required central executive resources, with manipulation but not maintenance being affected by a random word generation task (Mohr and Linden, 2005). These behavioral results support the assumption that our manipulation and maintenance paradigms tap different processes in working memory.

The half circles $\left(3.1 \times 1.34^{\circ}\right)$ were separated by $3.98^{\circ}$ center to center. The colors of the pairs of samples and the test were matched in brightness and saturation, according to the CIE 1931 standard observer for photopic luminous, $V \lambda$. The perceived combination of the two colors is located in the middle of the color space between the two colors [calculated in the CIE-1976 L* $\mathrm{u}^{\star} \mathrm{v}^{\star}$ color space; for details see Mohr and Linden (2005)]. In the spatial condition, the correct position of the half circle was along the line that bisects the angle between the two sample stimuli.

Off-line eye movement recordings were obtained in five subjects who also participated in the functional magnetic resonance imaging (fMRI) experiment, using an infrared eye tracker with a sampling rate of $100 \mathrm{~Hz}$ (Ober 2 digital eye movement registration system; Permobil Meditech, Timra, Sweden). No differences in eye movements of $>1^{\circ}$ of visual angle occurred between the color and spatial conditions ( $t$ test; criterion, $p<$ $0.05)$.

fMRI. fMRI data were acquired with a Siemens (Erlangen, Germany) 1.5 T Magnetom Vision MR tomograph using a gradient echo echoplanar imaging sequence (repetition time, $2000 \mathrm{~ms}$; echo time, 60; flip angle, $90^{\circ}$; field of view, $200 \times 200 \mathrm{~mm}^{2}$; voxel size, $3.13 \times 3.13 \times 5 \mathrm{~mm}^{3}$; 16 axial slices). In both experiments, subjects underwent three functional scans ( 365 volumes per scan, 10 volumes per trial, 36 trials per scan, pseudorandom order of color and spatial trials). A high-resolution T1weighted three-dimensional (3D) anatomical MR data set was used for coregistration.

Data analysis. Data were preprocessed with BrainVoyager QX (Brain Innovation, Maastricht, The Netherlands). Data preprocessing included slice scan time correction with the first scan time within a volume used as a reference for alignment by sinc interpolation, three-dimensional motion correction, spatial smoothing with an $8 \mathrm{~mm}$ Gaussian kernel (fullwidth at half-maximum), temporal highpass filtering to remove lowfrequency nonlinear drifts of three or fewer cycles per time course, and linear trend removal. Talairach transformation was performed for the complete set of functional data of each participant.

The statistical ANOVA of the blood oxygen level-dependent (BOLD) signal was based on the application of multiple regression analysis to time series of task-related functional activation (Friston et al., 1995). The general linear models (GLMs) of experiments 1 and 2 were computed from the $39 z$-normalized volume time courses, and the two experimental conditions (what and where) and three task phases encoding, delay, and retrieval (Fig. 1C) were considered effects of interest. The corresponding predictors, obtained by shifting an ideal box-car response by $4 \mathrm{~s}$ to account for the hemodynamic delay, were used to build the design matrix of the experiment. We chose the box-car model rather than a temporally smoothed hemodynamic response function to minimize overlap of predictors between task phases (Sack et al., 2002). 3D group statistic maps were generated by associating each voxel with the $F$ value corresponding to the specific set of predictors and calculated on the least mean square solution of the random-effects GLM.

Analysis was based on the random-effects GLM of the experiment with three (task phases: encoding, delay, and retrieval) times two (type of information: color, spatial) times two (processes: maintenance, manipulation) predictors. The obtained $\beta$ weights of each current predictor served as input for the second-level whole-brain random-effects analysis including a $2 \times 2$ factorial design for each task phase. Thus, the $\beta$ values of subjects were treated explicitly as realizations of the two withinsubjects factors "type of information" (level 1, what; level 2, where) and process (level 1, maintenance; level 2, manipulation). Linear contrasts what vs where and maintenance vs manipulation and interactions (level $1 \times 2$ ) between the experimental conditions were computed. Multisubject statistical maps were thresholded using the false discovery rate (FDR) (Genovese et al., 2002). Statistical results were visualized through projecting $3 \mathrm{D}$ maps on the inflated surface reconstruction of a template brain (courtesy of the Montreal Neurological Institute).

\section{Results}

\section{Behavioral data}

A $t$ test (dependent samples) showed no significant difference (criterion, $p<0.05$ ) in reaction times and accuracy between the task conditions of experiments 1 and 2 (reaction times, experiment 1 color vs spatial, $t=0.002, p=\mathrm{NS}$; experiment 2 color vs spatial, $t=1.9, p=\mathrm{NS}$; accuracy, experiment 1 color vs spatial, 
Table 1. Behavioral results of experiments 1 and 2

\begin{tabular}{|c|c|c|c|c|}
\hline & Color, mean/SE & Spatial, mean/SE & $t$ & $p$ \\
\hline \multicolumn{5}{|c|}{ Experiment 1, maintenance } \\
\hline RT & $1142,2 / 90$ & $1140,5 / 90.1$ & 0.002 & NS \\
\hline$\% \mathrm{C}$ & $69.5 / 3.6$ & $68.8 / 4.2$ & 0.1 & NS \\
\hline \multicolumn{5}{|c|}{ Experiment 2, manipulation } \\
\hline RT & $1094,6 / 49.3$ & $1005,5 / 57.5$ & 1.9 & NS \\
\hline$\% C$ & $69.1 / 1.9$ & $75 / 2.6$ & 1.8 & NS \\
\hline
\end{tabular}

Mean $( \pm$ SE) reaction time $(\mathrm{RT})$ and percent corrects $(\% \mathrm{C})$ for the conditions what and where in experiments 1 (maintenance) and 2 (manipulation). Comparison of RT and $\% \mathrm{C}$ between the two conditions $t$ test for dependent samples.

\section{Table 2. Comparison of behavioral data}

\begin{tabular}{lll}
\hline & $t$ & $p$ \\
\hline RT & & \\
$\quad$ Color & 0.4 & NS \\
Spatial & 1.4 & NS \\
C & & \\
Color & 0.29 & NS \\
Spatial & 1.4 & NS \\
\hline
\end{tabular}

Comparison of RT and C\% between experiments 1 and 2 in the two conditions with $t$ test for dependent samples ( $t$ and $p$ values).

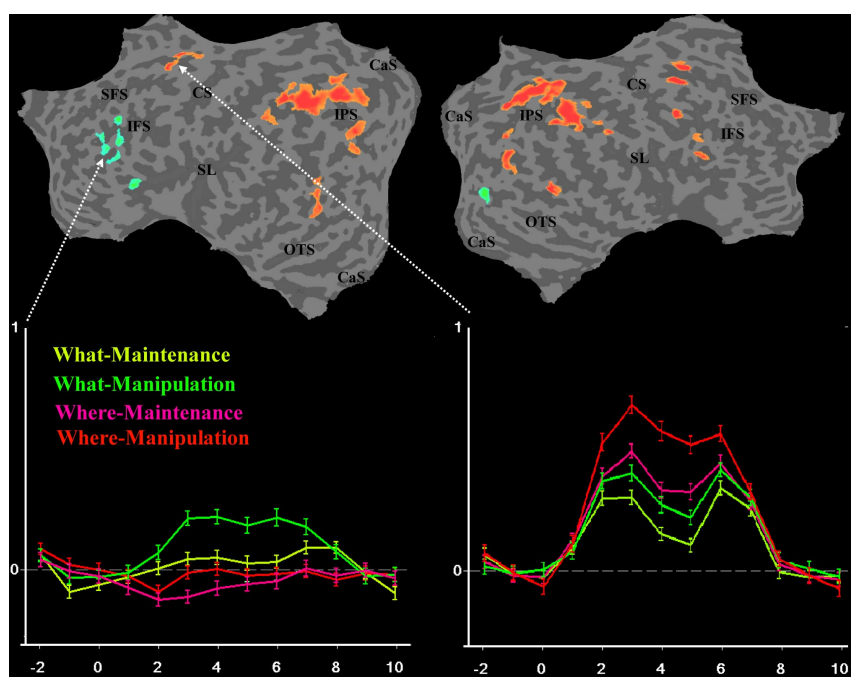

Figure 2. Random-effects GLM for what versus where in the delay. Below, Time courses of MFG (LH) and SFS (LH). Green, Color (what); red, spatial information (where); $q($ FDR) $<0.05$.

$t=0.1, p=\mathrm{NS}$; experiment 2 color vs spatial, $t=1.8, p=\mathrm{NS}$ ). No significant difference in reaction times and accuracy was observed between experiments 1 and 2 (reaction times: experiment 1 vs 2 color, $t=0.4, p=$ NS; experiment 1 vs 2 spatial, $t=1.4, p=$ NS; accuracy, experiment 1 vs 2 color, $t=0.29, p=$ NS; experiment 1 vs 2 spatial, $t=1.4, p=\mathrm{NS}$ ). Results are shown in Tables 1 and 2 .

\section{Neuroimaging data, linear contrast what versus where}

Cortical activation during the delay phase was separated into a more dorsal component for the spatial tasks [at the junction of superior frontal sulcus (SFS) and precentral sulcus (PCS) in both hemispheres] and a more ventral [along the inferior frontal sulcus (IFS), left hemisphere (LH) only] component for the color tasks (Fig. 2, Table 3) $[q($ FDR $)<0.05]$. As shown in the time course of the BOLD signal, the response in the left SFS/PCS is most pronounced for the condition "spatial (= WHERE) manipulation," followed by the condition "spatial maintenance." Conversely, the cluster around the left IFS shows the highest BOLD signal in the condition "color (= WHAT) manipulation." followed by "color maintenance."

Additional areas that were more active for the spatial conditions during delay included the parietal lobes and middle temporal gyrus bilaterally and right frontal regions (Fig. 2, Table 3). Outside the left VLPFC, only the right middle occipital gyrus showed higher delay activity in the color condition. Mostly similar patterns of dissociation between spatial and color processing were observed during encoding (Fig. $3 A)[q(\mathrm{FDR})<0.05]$ and retrieval (Fig. $3 B$, Table 4).

\section{Neuroimaging data, linear contrast maintenance versus manipulation}

To test for a possible frontal dorsoventral gradient for manipulation versus maintenance, as postulated by the process-specific theory (Owen et al., 1999), we computed a linear contrast between these predictors for all task phases, again thresholded at $q($ FDR $)<0.05$ (Fig. 4).

In the delay phase, frontal areas did not show any dorsoventral specialization for manipulation versus maintenance (Fig. 4). Conversely, significant fMRI signal increases for the manipulation tasks were observed in the middle frontal gyrus $(\mathrm{LH} / \mathrm{RH})$, insula $(\mathrm{LH})$, along the precentral gyrus $(\mathrm{LH} / \mathrm{RH})$ including the inferior frontal junction, and the caudal medial frontal gyrus (RH). Higher fMRI signal for the maintenance tasks was observed in the superior frontal gyrus (LH) and the rostral medial frontal gyrus ( $\mathrm{RH})$. Additional areas that were more active for the manipulation conditions during delay included the inferior parietal lobules $(\mathrm{LH} / \mathrm{RH})$ and the precuneus $(\mathrm{RH})$. For maintenance, additional areas were located in visual cortex and the cingulate. The analysis for the encoding and retrieval phases, like that for delay, did not reveal any dorsoventral process-specific dissociation of frontal activation (Table 5).

\section{Neuroimaging data, interaction material times process}

The interaction analysis between material (what and where) and process (maintenance and manipulation) $[q($ FDR $)<0.05]$ revealed no significant interaction effects at whole-brain level.

\section{Discussion}

One of the most striking aspects of human PFC activity during cognitive tasks seems to be its generality. A comparison of clusters of activation across studies yielded common areas along the IFS as being active for a wide range of cognitive tasks and stimulus types (Duncan and Owen, 2000). With the present study, we aimed to identify gradients for task and stimulus specificity within the PFC and adjacent areas by direct contrasts between different tasks (manipulation vs maintenance) and task-relevant stimulus attributes (color vs orientation). Such a gradient had been postulated for spatial versus object information on the basis of the connectivity and electrophysiological studies of monkey PFC (Constantinidis and Wang, 2004).

Our results provide evidence for specialization for stimulus attributes in frontal cortex. Activation along the IFS was more pronounced for the maintenance and manipulation of color. Higher activation for spatial compared with color WM was observed along the SFS/PCS. This activation cluster for spatial WM fell into the dorsal premotor cortex (dPMC; $x, y, z=-24,-8,61$; $28,-6,55$, center of mass) with some overlap with the putative 
human frontal eye field (FEF) in the right hemisphere (Koyama et al., 2004; Paus, 1996) $(x, y, z=-32,-2,46 ; 31,2,47)$ and the area specialized for spatial working memory identified by Courtney et al. (1998b) $(x, y, z=-31,-7,46 ; 27,-5$, 49). Our results support the suggestion that premotor areas and the FEF are not only involved in the voluntary generation of eye movements but also in the coding and rehearsal of spatial information for WM (Courtney et al., 1998b; Zarahn et al., 1999; Munk et al., 2002; Curtis and D’Esposito, 2003).

\section{The spatial tasks and premotor cortex}

The spatial condition of the present task was different from that of most previous studies, which required memorization of cue position rather than cue orientation. The spatial nature of the present angle transformation task was confirmed by an interference experiment with a classical spatial task. When the dual task involved a distance transformation (rather than the color manipulation), we did observe interference with the angle transformation task (Mohr and Linden, 2005). In terms of neural coding, cue position has been shown to be represented by the persistent discharge of frontal neurons that are specific for a part of visual space, their "memory field" (Constantinidis and Wang, 2004). The present spatial maintenance task might have been solved by such a mechanism as well, because the location of the end point of the base of the semicircle uniquely identified its orientation. However, the specific involvement of dorsal premotor cortex in the spatial tasks suggests additional potential mechanisms. Spatial orientation might be represented in activation of neurons in the FEF that code covert eye movements to the ends of the base. Alternatively, line orientation could be coded by directionally tuned cells in dPMC. Such cells have been shown to carry information about potential targets over the delay period of a reaching task (Cisek and Kalaska, 2005), but additional evidence will be needed to determine the relative contribution of premotor cortex to motor planning and active memory (Constantinidis and Procyk, 2004). The spatial manipulation condition of our experiment involved an angle transformation. This component of the task is thus related to classic mental rotation paradigms, for which activation in premotor areas has been shown with functional imaging (Richter et al., 2000).

The spatial condition activated a larger amount of brain tissue than the color task (Fig. 2). This greater activity might suggest that the spatial condition may be more complex. Alternatively, it is possible that a larger number of areas in the human brain have evolved to be specialized for spatial operations compared with the processing of colors. This interpretation is supported by the behavioral finding that both conditions are equal in difficulty.

\section{Ventrodorsal dissociations and maintenance of stimulus information}

The ventrodorsal dissociation of frontal activity in the monkey brain has been proposed to be analogous to that of the posterior visual pathways (Goldman-Rakic, 1987) with areas ventral to the sulcus principalis processing object-related information, and the dorsal areas processing spatial relations, locations, and motion. The similarity of our left ventral prefrontal activation focus to that found in previous human studies of face (Courtney et al., 1997) and object WM (Munk et al., 2002) suggests that the sub-

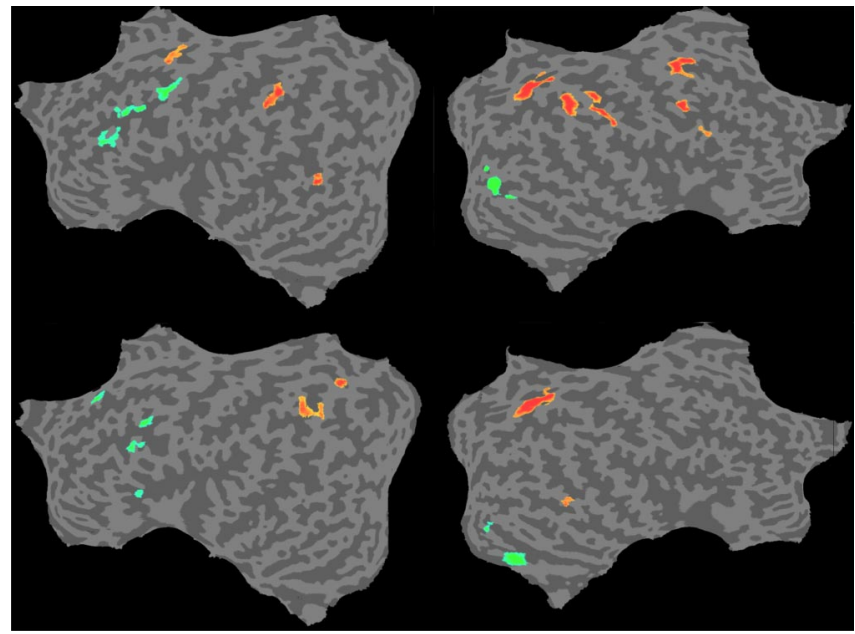

Figure 3. Random-effects GLM for what versus where in the encoding $(\boldsymbol{A})$ and retrieval $(\boldsymbol{B})$ phases. Green, Color (what); red, spatial information (where); $q($ FDR $)<0.05$.

division is indeed related to the functional segregation of the posterior visual processing streams. However, the segregation does not seem to follow the anatomical subdivision of lateral prefrontal cortex into a dorsal (DLPFC) and a ventral (VLPFC) part. The ventral activation clusters that we found for color processing included areas dorsal to the IFS [in the middle frontal gyrus (MFG)], whereas the dorsal cluster for spatial processing was posterior to the DLPFC in dorsal premotor areas. It is important to note that the human homolog of the monkey sulcus principalis seems to be the intermediate frontal sulcus (Petrides and Pandya, 1999), which shows large individual variation in folding in the MFG. It is therefore not possible to locate the cluster activated for our color task unambiguously into the human analog of the VLPFC.

The prefrontal region in the IFS (BA 46/45) might be the site where the color information is actually stored by neurons with selective delay-related activity. Alternatively, it might control the rehearsal of color information through a cross talk with more posterior visual areas. Our data are compatible with both accounts. Activity in occipitotemporal areas with a potential overlap with the color-specific area V4 (McKeefry and Zeki, 1997) was in fact observed during all periods of our task (Figs. 2, 3). Petrides $(1994,1996)$ proposed for the macaque that areas 46 and 9/46 are specialized regions where stimuli that are maintained in posterior association cortical areas can be recoded in an abstract form for the purpose of the monitoring of expected acts or events. In the case of our paradigm, the color information might have been recoded by 
Table 4. GLM encoding/retrieval what versus where

\begin{tabular}{|c|c|c|c|c|c|c|}
\hline $\begin{array}{l}\text { Area } \\
\text { (anatomical/Brodmann) }\end{array}$ & Hem. & Predictor & $x$ & $y$ & $z$ & $\begin{array}{l}\text { Cluster size } \\
\left(\mathrm{mm}^{3}\right)\end{array}$ \\
\hline \multicolumn{7}{|l|}{ Encoding } \\
\hline IFS/46 & $\mathrm{LH}$ & Color & -42 & 26 & 22 & 1233 \\
\hline $\mathrm{PCS} / 4$ & $\mathrm{LH}$ & Color & -47 & -8 & 48 & 886 \\
\hline Middle frontal gyrus/6 & $\mathrm{LH}$ & Spatial & -23 & -10 & 56 & 1349 \\
\hline Inferior parietal lobule/40 & $\mathrm{LH}$ & Spatial & -35 & -41 & 46 & 1750 \\
\hline Middle temporal gyrus/37 & $\mathrm{LH}$ & Spatial & -51 & -60 & 1 & 598 \\
\hline Precentral gyrus/6 & $\mathrm{RH}$ & Spatial & 28 & -10 & 54 & 1606 \\
\hline Inferior frontal gyrus/9 & $\mathrm{RH}$ & Spatial & 55 & 5 & 31 & 2123 \\
\hline Inferior parietal lobule/40 & $\mathrm{RH}$ & Spatial & 49 & -33 & 42 & 4323 \\
\hline Precuneus/7 & $\mathrm{RH}$ & Spatial & 17 & -64 & 49 & 1899 \\
\hline Middle occipital gyrus/18 & $\mathrm{RH}$ & Color & 27 & -84 & 2 & 2527 \\
\hline \multicolumn{7}{|l|}{ Retrieval } \\
\hline Superior frontal gyrus/8 & $\mathrm{LH}$ & Color & -16 & 35 & 44 & 516 \\
\hline Inferior frontal gyrus/9 & $\mathrm{LH}$ & Color & -40 & 6 & 31 & 1844 \\
\hline Claustrum & $\mathrm{LH}$ & Color & -30 & 31 & 7 & 1143 \\
\hline Precuneus/7 & $\mathrm{LH}$ & Spatial & -19 & -59 & 49 & 1626 \\
\hline Precuneus/7 & $\mathrm{RH}$ & Spatial & 19 & -64 & 51 & 2942 \\
\hline Middle temporal gyrus/37 & $\mathrm{RH}$ & Spatial & 59 & -45 & -5 & 323 \\
\hline Parahippocampal gyrus/19 & $\mathrm{RH}$ & Color & 23 & -44 & -2 & 953 \\
\hline Lingual gyrus/18 & $\mathrm{RH}$ & Color & 19 & -83 & 0 & 1377 \\
\hline
\end{tabular}

Centers of mass of significant contrasts between what and where conditions (encoding and retrieval). Hem., Hemisphere; LH, left hemisphere; RH, right hemisphere; Predictor, condition with the stronger BOLD response; $x, y, z$, Talairach coordinates.

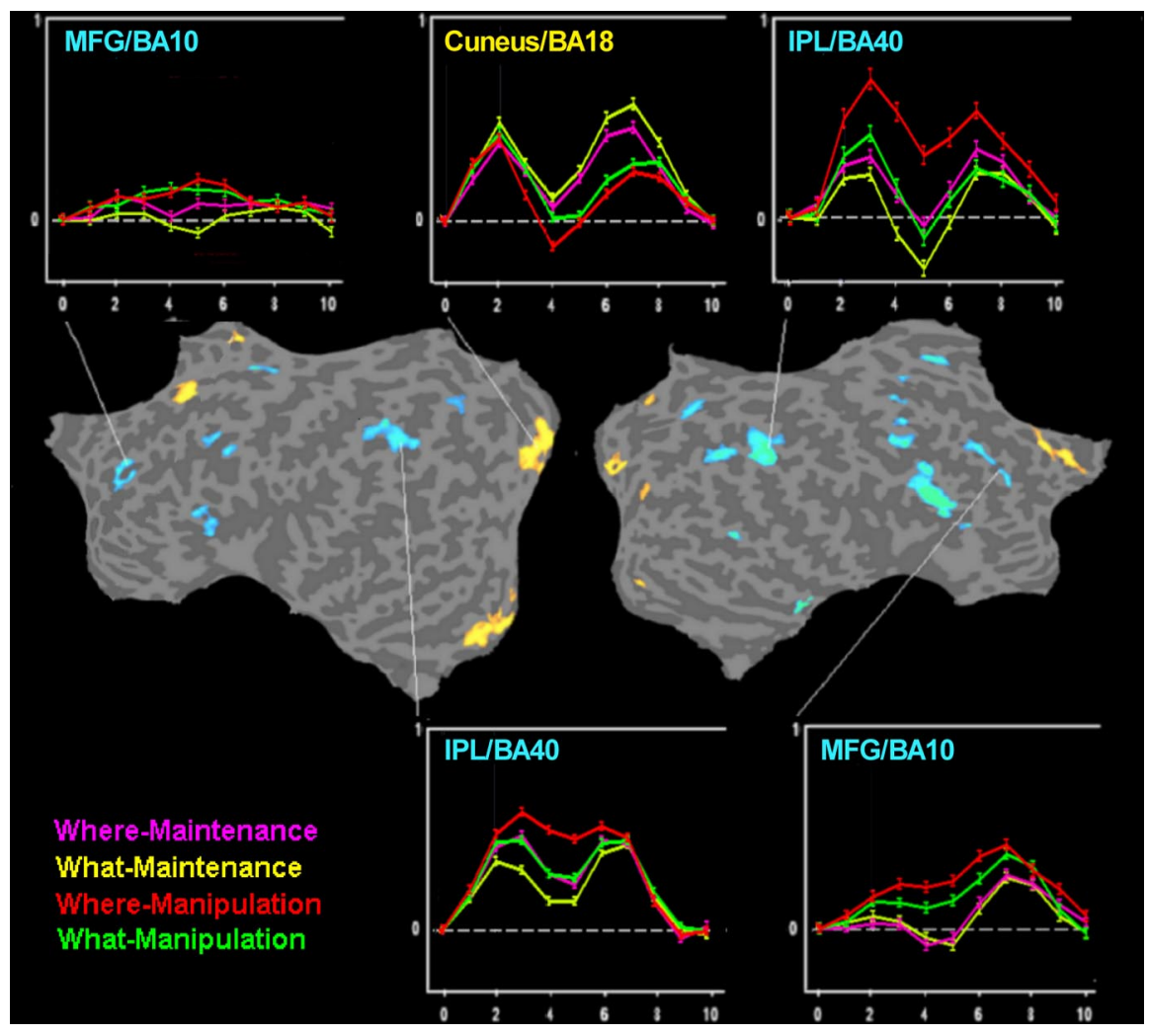

Figure 4. Random-effect GLM comparing maintenance versus manipulation for the color and spatial conditions during delay. Yellow, Areas activated for maintenance; blue, areas activated for manipulation; $q(\mathrm{FDR})<0.05$. No ventrodorsal segregation of the PFC according to the kind of process in the delay phase. Bottom and top, Time courses of selected brain regions.

activity of the neurons around the IFS. This representation may be used for higher cognitive functions like task monitoring or stimulus manipulation or to coordinate the rehearsal of task relevant color information stored by the occipitotemporal cortex.

Our data also suggest an additional subdivision of prefrontal specialization into areas for storage and rehearsal of verbal and visual information. The area of activation for the color tasks is different from the area for subvocal rehearsal (Logie et al., 2003), which makes it unlikely that the observed dissociation of frontal cortex is based on a verbal-spatial distinction (center of mass of the cluster for subvocal rehearsal: $x, y, z=-50,16,18$; for color, $x, y$, $z=-40,29,16)$.

\section{The manipulation network}

The comparison between maintenance and manipulation revealed higher activity in a frontoparietal network for the manipulation tasks. The activated frontal areas related to manipulation (BA 10, 6, 44) are systematically activated by a large range of executive tasks, suggesting their involvement in general executive processes (Collette and Van der Linden, 2002). We also demonstrate bilateral activation of parietal regions (inferior parietal lobule BA 40, Precuneus BA 7), which again conforms to findings on different kinds of executive tasks (Collette et al., 1999). The model of Curtis and D'Esposito (2003) suggests that the DLPFC (BA 46, 9) contains a domainindependent area that is primarily involved in the top-down control of working memory, independent of the type of stored material. In our study, regions in the middle frontal gyrus ( $\mathrm{RH}, \mathrm{BA} 9$; $\mathrm{LH}$, BA 9, 10) were more active during manipulation than during maintenance of information, regardless of the kind of material. This activation might be a correlate of the higher demand of top down control during the manipulation than during the maintenance of internal representations. This finding of predominantly anterior PFC activation during manipulation fits recent models of the hierarchical architecture of cognitive control. Rostral lateral PFC and frontopolar cortex have been postulated to be engaged in cognitive branching, the organization of sequential subprocesses (Koechlin et al., 2003). Because of the dual requirement of encoding and transformation of the visual material, demand on this function was higher during the manipulation than during the maintenance conditions of our paradigm.

Cognitive control in WM has often been linked to areas of rostral PFC, but there is also evidence for the involvement of more posterior frontolateral regions around the junction of the inferior frontal gyrus and the inferior precentral sulcus [inferior frontal junction area (IFJ) (Derrfuss et al., 2004)]. These authors found that among other regions, the IFJ $(x, y, z=-38,2,32 ; x, y, z=47,8$, 30 ) and the anterior insular were commonly activated for three different central executive/cognitive control tasks (task switching, manual Stroop task, and a verbal n-back task). In the present 
study, we also found bilaterally activated clusters along the precentral sulcus, including the IFJ ( $x, y, z=-40,2,39 ; x, y$, $z=47,3,36$ ), and the insula for the manipulation tasks. Our data thus provide additional evidence for a role of the IFJ in cognitive control processes. The design of our study was intended to impose a higher demand on cognitive control in the manipulation condition. Considering the finding of the behavioral study that only the manipulation tasks were disrupted by a concurrent central executive task (Mohr and Linden, 2005), we would conclude that this aim was indeed achieved by the present paradigm.

The higher maintenance-related activity in visual cortex during delay is probably a result of the more sluggish return to baseline after higher encoding activation than in the manipulation conditions (Fig. 4). However, the maintenance effect in the cingulate (BA 31) reflects a genuine activation increase, as opposed to a slight suppression during manipulation. This part of the cingulate and the functionally connected BA 8 and BA 9 have been implicated in the default-mode network (Greicius et al., 2003; Fox et al., 2005), which is classically deactivated during demanding tasks. The difference in the cingulate, the superior frontal gyrus (BA 8), and the medial frontal gyrus (BA 9) between activation during maintenance and deactivation during manipulation might reflect the need to suppress concurrent cognitive processes particularly in the manipulation task.

In summary, we demonstrate that the functional organization of the neural system that supports visual working memory has both content- and task-specific elements. The parallel processing of color and spatial information is supported by specialized areas in frontal, parietal, and visual cortex. In the left frontal cortex, this dissociation followed a dorsoventral gradient, with dorsal premotor cortex more active during spatial and a region along the IFS during color working memory, regardless of task. Furthermore, we also found brain areas that specialized in task regardless of content. For manipulation, these included a frontoparietal network commonly implicated in cognitive control. Our data are thus compatible with models of multiple levels of specialization in frontal cortex.

\section{References}

Baddeley AD, Hitch G (1974) Working memory. In: Recent advances in learning and motivation (Bower GA, ed), pp 47-90. New York: Academic. Cisek P, Kalaska JF (2005) Neural correlates of reaching decisions in dorsal premotor cortex: specification of multiple direction choices and final selection of action. Neuron 45:801-814.
Table 5. GLM maintenance versus manipulation

\begin{tabular}{|c|c|c|c|c|c|c|}
\hline Area (anatomical/ Brodmann) & Hem. & Predictor & $x$ & $y$ & $z$ & $\begin{array}{l}\text { Cluster size } \\
\left(\mathrm{mm}^{3}\right)\end{array}$ \\
\hline \multicolumn{7}{|l|}{ Delay } \\
\hline Superior frontal gyrus/8 & LH & Maint. & -10 & 37 & 49 & 8361 \\
\hline Middle frontal gyrus/10 & LH & Manip. & -31 & 45 & 21 & 913 \\
\hline Precentral sulcus, IFJ/6 & LH & Manip. & -40 & 2 & 39 & 1089 \\
\hline Insula/13 & LH & Manip. & -36 & 19 & 11 & 1076 \\
\hline Precentral gyrus/6 & LH & Manip. & -22 & -11 & 64 & 406 \\
\hline Cingulate/31 & LH & Maint. & -9 & -11 & 47 & 211 \\
\hline Inferior parietal lobule/40 & LH & Manip. & -34 & -46 & 43 & 3037 \\
\hline Cuneus/18 & LH & Maint. & -6 & -71 & 16 & 9421 \\
\hline Medial frontal gyrus/9 & RH & Maint. & 2 & 49 & 19 & 4526 \\
\hline Middle frontal gyrus $/ 9$ & RH & Manip. & 40 & 38 & 35 & 4141 \\
\hline Inferior frontal gyrus/44 and insula /13 & $\mathrm{RH}$ & Manip. & 45 & 14 & 16 & 6273 \\
\hline Precentral sulcus, IFJ/6 & RH & Manip. & 47 & 3 & 36 & 1890 \\
\hline Medial frontal gyrus/6 & $\mathrm{RH}$ & Manip. & 6 & 6 & 51 & 1006 \\
\hline Inferior parietal lobule/40 & $\mathrm{RH}$ & Manip. & 42 & -46 & 43 & 5187 \\
\hline Precuneus/7 & RH & Manip. & 1 & -66 & 46 & 2178 \\
\hline Middle temporal gyrus/37 & $\mathrm{RH}$ & Manip. & 58 & -52 & 0 & 279 \\
\hline Posterior cingulate/31 & $\mathrm{RH}$ & Maint. & 9 & -50 & 24 & 219 \\
\hline Cuneus/18 & $\mathrm{RH}$ & Maint. & 1 & -75 & 20 & 2201 \\
\hline Cuneus/18 & $\mathrm{RH}$ & Maint. & 19 & -83 & 25 & 475 \\
\hline Lingual gyrus/18 & $\mathrm{RH}$ & Maint. & 12 & -69 & 0 & 289 \\
\hline \multicolumn{7}{|l|}{ Encoding } \\
\hline Medial frontal gyrus/9 & $\mathrm{RH}$ & Maint. & 7 & 46 & 32 & 4099 \\
\hline Superior frontal gyrus/6 & RH & Maint. & 19 & 25 & 52 & 542 \\
\hline Precentral gyrus/6 & $\mathrm{RH}$ & Manip. & 35 & -12 & 59 & 1290 \\
\hline Precentral gyrus/9 & $\mathrm{RH}$ & Manip. & 43 & 6 & 33 & 1700 \\
\hline Inferior parietal lobule/40 & $\mathrm{RH}$ & Manip. & 40 & -43 & 42 & 2665 \\
\hline Precuneus/7 & $\mathrm{RH}$ & Manip. & 14 & -69 & 44 & 6848 \\
\hline Cuneus/18 & RH & Maint. & 26 & -95 & 2 & 1376 \\
\hline Cuneus/17 & RH & Manip. & 11 & -77 & 11 & 855 \\
\hline Medial frontal gyrus/8 & LH & Maint. & -7 & 40 & 39 & 4357 \\
\hline Superior frontal gyrus/8 & LH & Maint. & -20 & 20 & 40 & 3286 \\
\hline Middle frontal gyrus/9 & LH & Manip. & -34 & 32 & 29 & 326 \\
\hline Precentral gyrus/6 & LH & Manip. & -40 & 2 & 27 & 751 \\
\hline Inferior parietal lobule/40 & LH & Manip. & -58 & -26 & 33 & 283 \\
\hline Inferior parietal lobule/40 & LH & Manip. & -37 & -45 & 54 & 1035 \\
\hline Precuneus/7 & LH & Manip. & -13 & -70 & 40 & 2465 \\
\hline Cingulate gyrus/31 & LH & Maint. & -6 & -39 & 32 & 584 \\
\hline Inferior parietal lobule/40 & LH & Maint. & -47 & -59 & 38 & 4142 \\
\hline Cuneus/18 & LH & Maint. & -4 & -79 & 19 & 200 \\
\hline Inferior occipital gyrus/18 & LH & Maint. & -33 & -84 & -6 & 440 \\
\hline Lingual gyrus/18 & LH & Manip. & -9 & -78 & 4 & 1212 \\
\hline \multicolumn{7}{|l|}{ Retrieval } \\
\hline $\mathrm{IFS} / 9$ & LH & Manip. & -36 & 27 & 27 & 5653 \\
\hline Middle frontal gyrus/10 & LH & Manip. & -40 & 40 & 23 & 2775 \\
\hline Middle frontal gyrus/9 & LH & Maint. & -25 & 18 & 47 & 1020 \\
\hline Medial frontal gyrus/ 6 & LH & Maint. & -2 & -2 & 53 & 1513 \\
\hline Middle frontal gyrus/6 & LH & Maint. & -32 & -4 & 51 & 237 \\
\hline Superior parietal lobule/7 & LH & Manip. & -32 & -47 & 60 & 2531 \\
\hline Superior parietal lobule/7 & LH & Maint. & -26 & -54 & 43 & 609 \\
\hline Cingulate gyrus & LH & Maint. & -9 & -42 & 40 & 569 \\
\hline Lingual gyrus/17 & LH & Manip. & -14 & -93 & -7 & 1256 \\
\hline Lingual gyrus/18 & LH & Maint. & -11 & -66 & 3 & 21342 \\
\hline Posterior cingulate/30 & LH & Maint. & -31 & -74 & 9 & 1942 \\
\hline
\end{tabular}

Centers of mass of significant contrasts between manipulation and maintenance tasks. Maint., Maintenance; Manip., manipulation; Hem., hemisphere; LH left hemisphere; RH, right hemisphere; Predictor, condition with the stronger BOLD response; $x, y, z$, Talairach coordinates. 
Constantinidis C, Wang XJ (2004) A neural circuit basis for spatial working memory. Neuroscientist 10:553-565.

Courtney SM, Ungerleider LG, Keil K, Haxby JV (1997) Transient and sustained activity in a distributed neural system for human working memory. Nature 386:608-611.

Courtney SM, Petit L, Haxby JV, Ungerleider LG (1998a) The role of prefrontal cortex in working memory: examining the contents of consciousness. Philos Trans R Soc Lond B Biol Sci 353:1819-1828.

Courtney SM, Petit L, Maisog J, Ungerleider L, Haxby J (1998b) An area specialized for spatial working memory in human frontal cortex. Science 279:1347-1351.

Curtis CE, D’Esposito M (2003) Persistent activity in the prefrontal cortex during working memory. Trends Cogn Sci 7:415-423.

Derrfuss J, Brass M, von Cramon DY (2004) Cognitive control in the posterior frontolateral cortex: evidence from common activations in task coordination, interference control and working memory. NeuroImage 23:604-612.

D’Esposito M, Postle BR, Ballard D, Lease J (1999) Maintenance versus manipulation of information held in working memory: an event-related fMRI study. Brain Cogn 41:66-86.

Duncan J, Owen A (2000) Common regions of the human frontal lobe recruited by diverse cognitive demands. Trends Neurosci 23:475-483.

Elliott R, Dolan RJ (1998) The neural response in short-term visual recognition memory for perceptual conjunctions. NeuroImage 7:14-22.

Fletcher P, Henson RNA (2001) Frontal lobes and human memory. Insights from functional neuroimaging. Brain 124:849-881.

Fox MD, Snyder AZ, Vincent JL, Corbetta M, Van Essen DC, Raichle ME (2005) The human brain is intrinsically organized into dynamic, anticorrelated functional networks. Proc Natl Acad Sci USA 102:9673-9678.

Friston KJ, Holmes AP, Worsley KJ, Poline JP, Frith CD, Frackowiak RSJ (1995) Statistical parametric maps in functional imaging: a general linear approach. Hum Brain Mapp 2:189-210.

Genovese CR, Lazar NA, Nichols T (2002) Thresholding of statistical maps in functional neuroimaging using the false discovery rate. NeuroImage 15:870-878.

Goldman-Rakic PS (1987) Circuitry of primate prefrontal cortex and regulation of behavior by representational memory. In: Handbook of physiology (Mountcastle F, Plum F, eds), pp 373-417. Washington, DC: American Physiology Society.

Greicius MD, Krasnow B, Reiss AL, Menon V (2003) Functional connectivity in the resting brain: a network analysis of the default mode hypothesis. Proc Natl Acad Sci USA 100:253-258.

Hoshi E, Tanji J (2004) Area-selective neuronal activity in the dorsolateral prefrontal cortex for information retrieval and action planning. Neurophysiology 91:2707-2722.

Ishihara MD (2001) Ishihara's tests for color deficiency. Tokyo: Kanehara.

Koechlin E, Ody C, Kounelher F (2003) The architecture of cognitive control in the human prefrontal cortex. Science 302:1181-1185.

Koyama M, Hasegawa I, Osada T, Adachi Y, Nakahara K, Miyashita Y (2004) Functional magnetic resonance imaging of macaque monkeys performing visually guided saccade tasks: comparison of cortical eye fields with humans. Neuron 41:795-807.

Logie RH, Venneri A, Della Sala S, Redpath TW, Marshall I (2003) Brain activation and the phonological loop: the impact of rehearsal. Brain Cogn 53:293-296.

McKeefry DJ, Zeki S (1997) The position and topography of the human color centre as revealed by functional magnetic resonance imaging. Brain 120:2229-2242.

Mohr HM, Linden DEJ (2005) Separation of the systems for color and spatial manipulation in working memory revealed by a dual task procedure. J Cogn Neurosci 17:355-366.

Mohr HM, Morawetz C, Goebel R, Singer W, Linden DEJ (2003) Ventraldorsal segregation of sensory and prefrontal cortical activity related to color and spatial manipulation. Soc Neurosci Abstr 29:343.2.

Munk MH, Linden DE, Muckli L, Lanfermann H, Zanella FE, Singer W, Goebel R (2002) Distributed cortical systems in visual short-term memory revealed by event-related functional magnetic resonance imaging. Cereb Cortex 12:866-876.

Owen AM, Herrod NJ, Menon DK, Clark JC, Downey SP, Carpenter TA, Minhas PS, Turkheimer FE, Williams EJ, Robbins TW, Sahakian BJ, Petrides M, Pickard JD (1999) Redefining the functional organization of working memory processes within human lateral prefrontal cortex. Eur J Neurosci 11:567-574.

Paus T (1996) Location and function of the human frontal eye-field: a selective review. Neuropsychologia 34:475-483.

Petrides M (1994) Frontal lobes and working memory: evidence from investigations of the effects of cortical excisions in nonhuman primates. In: Handbook of neuropsychology, Vol 9 (Boller F, Grafman J, eds), pp 5982. Amsterdam: Elsevier.

Petrides M (1996) Specialized systems for the processing of mnemonic information within the primate frontal cortex. Philos Trans R Soc Lond B Biol Sci 351:1455-1462.

Petrides M (2000) The role of the mid-dorsolateral prefrontal cortex in working memory. Exp Brain Res 133:44-54.

Petrides M, Pandya DN (1999) Dorsolateral prefrontal cortex: comparative cytoarchitectonic analysis in the human and the macaque brain and corticocortical connection patterns. Eur J Neurosci 11:1011-1036.

Rämä P, Poremba A, Sala JB, Yee L, Malloy M, Mishkin M, Courtney SM (2004) Dissociable functional cortical topographies for working memory maintenance of voice identity and location. Cereb Cortex 14:768-780.

Richter W, Somorjai R, Summers R, Jarmasz M, Menon RS, Gati JS, Georgopoulos AP, Tegeler C, Ugurbil K, Kim SG (2000) Motor area activity during mental rotation studied by time-resolved single-trial fMRI. J Cogn Neurosci 12:310-320.

Rowe JB, Passingham RE (2001) Working memory for location and time: activity in prefrontal area 46 relates to selection rather than maintenance in memory. NeuroImage 14:77-86.

Rowe JB, Toni I, Josephs O, Frackowiak RS, Passingham RE (2000) The prefrontal cortex: response selection or maintenance within working memory? Science 288:1656-1660.

Sack AT, Sperling JM, Prvulovic D, Formisano E, Goebel R, Di Salle F, Dierks $\mathrm{R}$, Linden DE (2002) Tracking the mind's image in the brain II: transcranial magnetic stimulation reveals parietal asymmetry in visuospatial imagery. Neuron 35:195-204.

Sala JB, Rämä P, Courtney SM (2003) Functional topography of a distributed neural system for spatial and nonspatial information in working memory. Neuropsychologia 41:341-356.

Yee TSL, Sala JB, Courtney SM (2003) Differential dorsal and ventral activation in shape versus color working memory. Soc Neurosci Abstr 29:287.16.

Zarahn E, Aguirre GK, D’Esposito M (1999) Temporal isolation of the neural correlates of spatial mnemonic processing with fMRI. Brain Res Cogn Brain Res 7:255-268. 